\title{
Thyroid and Heart: Severe Three Vessel Coronary Artery Disease in a Middle-Aged Female with Hypothyroidism
}

Ramsha Muneer ${ }^{1}$, Syed Arsalan Ahmed Naqvi ${ }^{1}$, Owais Gul ${ }^{1}$, Syed Danish H. Zaidi ${ }^{2}$, Mudassir Iqbal Dar ${ }^{2}$

1. Internal Medicine, Dow University of Health Sciences, Karachi, PAK 2. Cardiac Surgery, Civil Hospital Karachi, Karachi, PAK

Corresponding author: Owais Gul, owaisgul96@yahoo.com

\begin{abstract}
Hypothyroidism is a common medical condition. The low metabolic state in hypothyroidism leads to significant cardiovascular and hemodynamic changes. Hypothyroidism is associated with heart failure, diastolic hypertension, atherosclerosis, coronary artery disease (CAD), and decreased insulin sensitivity. Similarly, the administration of levothyroxine worsens the cardiovascular disease by establishing a supplydemand mismatch. Here, we present a case of a 45 -year-old woman with hypothyroidism who presented to us with exertional chest pain and later got diagnosed with severe three-vessel disease. Coronary artery bypass grafting $(\mathrm{CABG})$ surgery was planned after the establishment of euthyroid state.
\end{abstract}

Categories: Cardiology, Endocrinology/Diabetes/Metabolism, Internal Medicine Keywords: hypothyroidism, three vessel disease, cabg

\section{Introduction}

Thyroid hormones play a major role in regulating the metabolic physiology of the human body and exert significant effects on the cardiovascular system. Hypothyroidism is associated with various cardiometabolic changes. These changes include impaired diastolic function, decreased cardiac contractility, endothelial dysfunction, decreased endothelial nitric oxide production, decreased arterial compliance, and decreased vascular smooth muscle relaxation. This low metabolic state in hypothyroidism leads to heart failure, diastolic hypertension, atherosclerosis, and sometimes coronary artery disease (CAD) [1]. Similarly, decreased insulin sensitivity due to the downregulation of glucose transporters, insulin resistance, or decreased insulin secretion can also be associated with hypothyroidism [2]. We present a case of a 45-yearold woman having a three-vessel disease with underlying severe hypothyroidism, non-insulin dependent diabetes mellitus, and hypertension, who presented to us with typical chest pain.

Received 10/23/2019 Review began 11/03/2019 Review ended 11/05/2019 Published 11/08/2019

\section{() Copyright 2019}

Muneer et al. This is an open access article distributed under the terms of the Creative Commons Attribution License CC-BY 3.0., which permits unrestricted use, distribution, and reproduction in any medium, provided the original author and source are credited.

\section{Case Presentation}

A 45-year-old married woman presented to the emergency department with the primary complaints of chest pain and palpitations for a week. She was diagnosed with hypothyroidism five years back for which she had been on thyroid replacement therapy. However, she was diagnosed with hypertension and diabetes mellitus a couple of years back. She reported that she had been experiencing chest pain while performing household activities accompanied by severe shortness of breath, paroxysmal nocturnal dyspnea, and occasional episodes of nausea. The pain was sudden in onset, throbbing in character, radiating to both arms, and was relieved by sublingual nitroglycerin. She denied any symptoms of accompanying cough, syncope, joint pain, or edema. Furthermore, her history ruled out any previous trauma and symptoms related to congenital heart disease, congestive cardiac failure, or myocardial infarction. Her past medical and surgical history was insignificant. Her systemic review, which was otherwise unremarkable, however, indicated hair loss, significant weight gain, and infrequent bowel habits. There was no history of tobacco, alcohol, or recreational drug use. Her family history was insignificant for cardiovascular disorders or autoimmune disorders of any sort. She had been taking losartan $25 \mathrm{mg}$ /day and oral hypoglycemic drugs for hypertension and diabetes mellitus, respectively.

On physical examination, she was fully alert and oriented. The patient was afebrile with a regular pulse rate of 75 beats per minute (BPM) and a blood pressure of 110/70 millimeter of mercury (mmHg). Jaundice, lymphadenopathy, cyanosis, and edema were not found. Neck examination was unremarkable except for a mildly enlarged thyroid. Cardiac examination did not reveal any abnormal heart sounds or raised jugular venous pulse. Lungs were clear to auscultation bilaterally with a respiratory rate of 20 breaths per minute. The abdominal and neurological examinations were insignificant. Electrocardiography (ECG) showed sinus rhythm. The patient was admitted for further evaluation.

Complete blood count (CBC), erythrocyte sedimentation rate (ESR), C reactive protein (CRP), coagulation profile, liver function tests (LFTs), blood urea nitrogen (BUN), creatinine (Cr), and basic metabolic panel (BMP) were within normal limits. Cardiac Troponin-I (cTnI) and thyroid stimulating hormone (TSH) were 


\section{Cureus}

elevated with values of 5.19 nanograms/milliliters $(\mathrm{ng} / \mathrm{ml})$ and 70 milli international units per liter (mIU/L), respectively. Similarly, HbA1c was also found elevated at $8.8 \%$.

Non-invasive imaging tests were performed, which included echocardiography and neck ultrasound. The echocardiography revealed segmental posterior wall motion abnormality and grade one diastolic dysfunction. The neck ultrasound did not reveal any significant findings. Her thyroid scan was normal. Later, cardiac angiography was done, which showed severe three-vessel disease in a right dominant heart circulation (Figures 1-3).

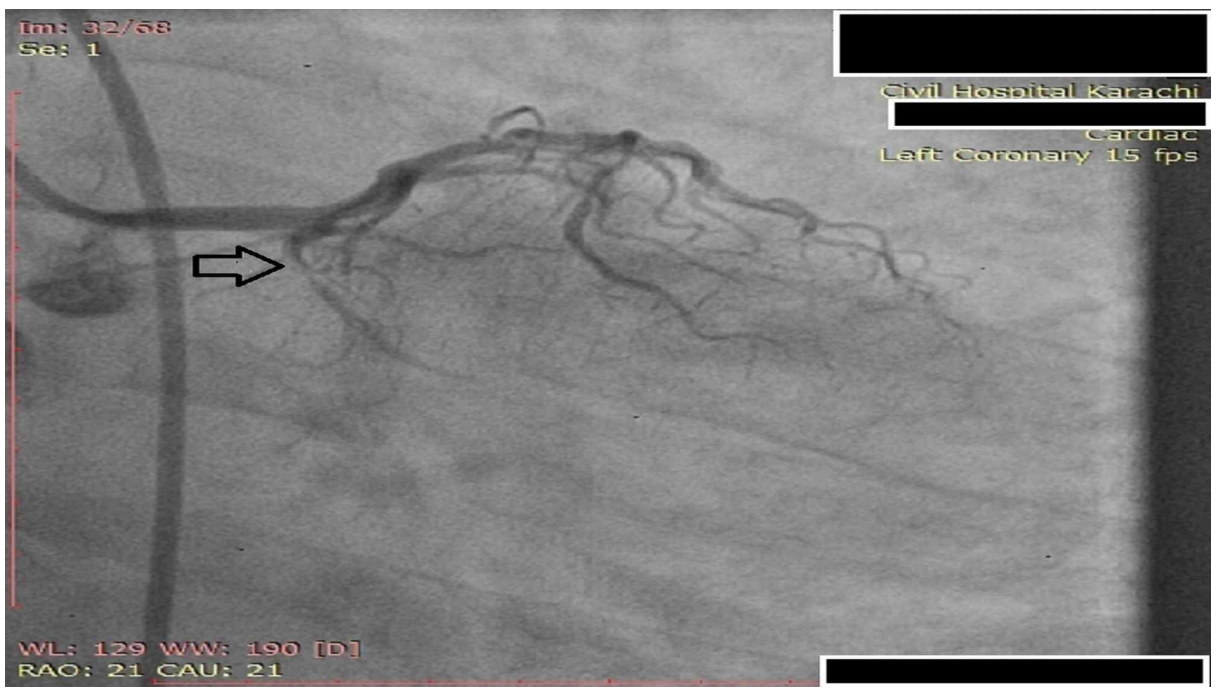

FIGURE 1: Angiogram of LCX coronary artery showing severe (90-100\%) distal segment stenosis

LCX: Left circumflex

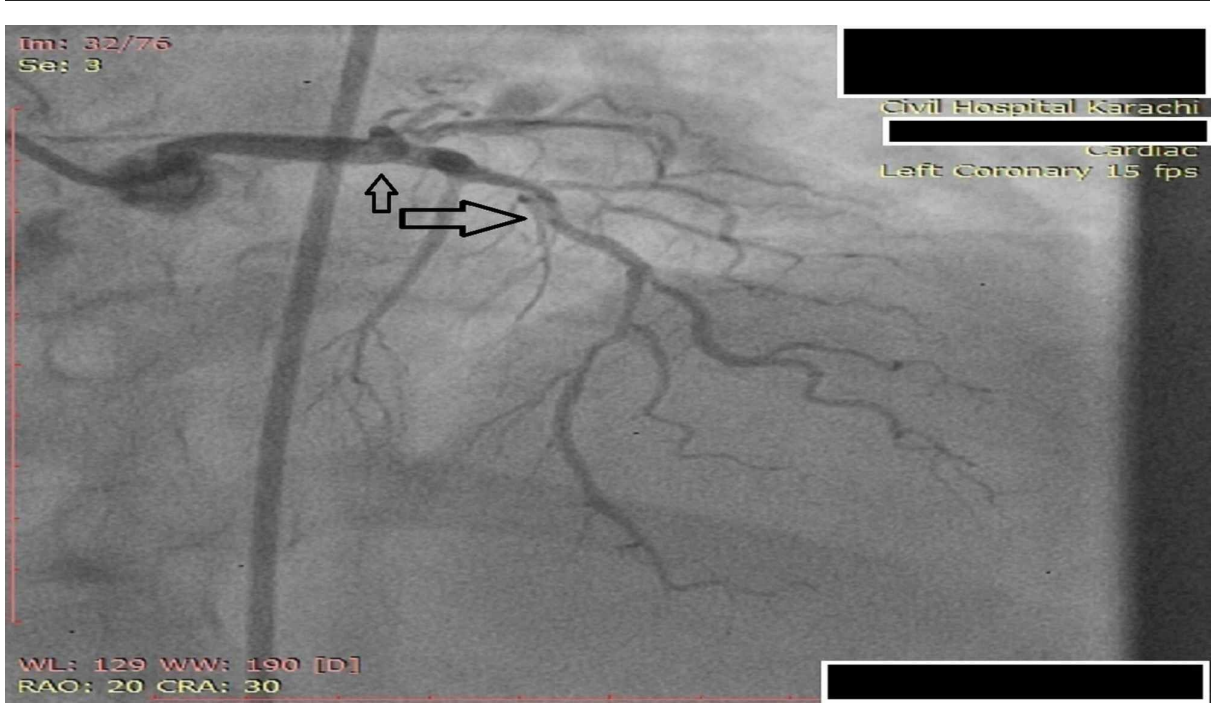

FIGURE 2: Angiogram of LAD coronary artery showing moderate $\mathbf{( 5 0 \% )}$ proximal segment and severe $(70 \%)$ mid-segment stenosis

LAD: Left anterior descending 


\section{Cureus}

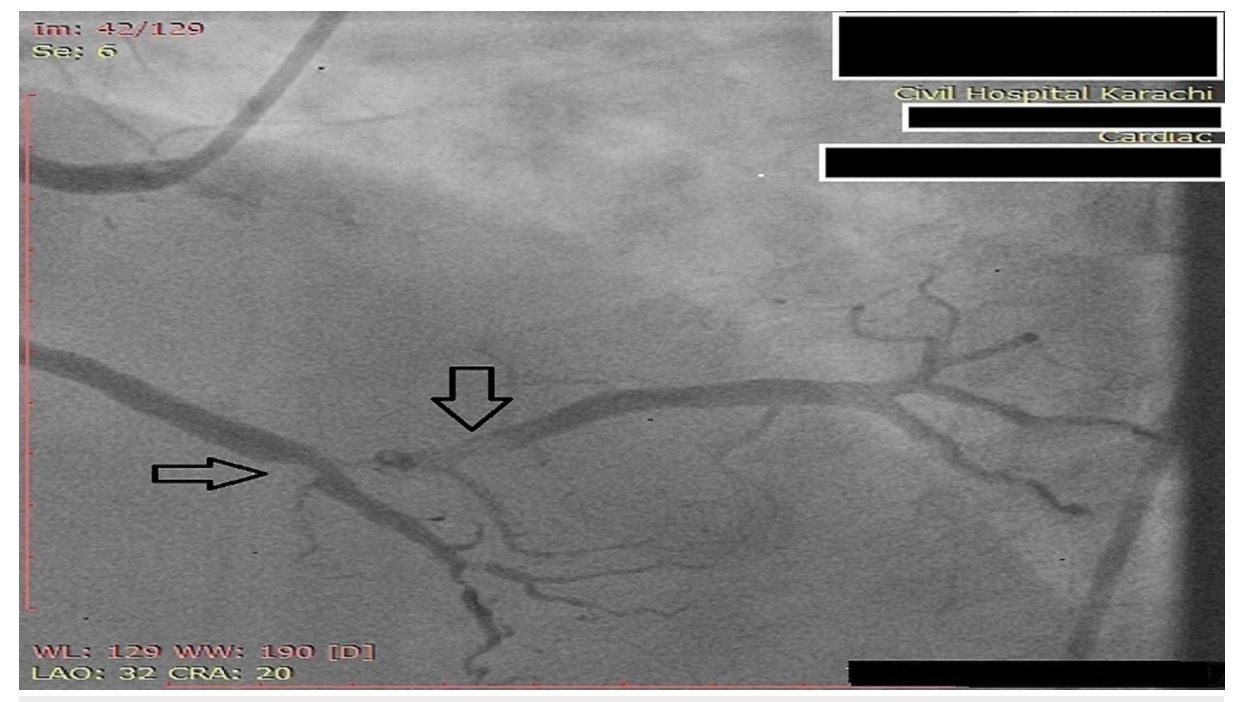

FIGURE 3: Angiogram of RCA showing severe (80-90\%) blockage with complete stenosis of PDA

RCA: Right coronary artery, PDA: Posterior descending artery

Coronary artery bypass grafting (CABG) surgery was planned after the establishment of euthyroid state. She was started on multiple drugs, which included aspirin $75 \mathrm{mg} /$ day, metoprolol $25 \mathrm{mg}$ twice a day, ranitidine 5 $\mathrm{mg} / \mathrm{day}$, thyroxine $500 \mathrm{mg} / \mathrm{day}$, and gliclazide $30 \mathrm{mg}$ twice a day. The patient was advised to return for surgery after a week; however, she did not come back.

\section{Discussion}

Hypothyroidism is a commonly encountered clinical condition that affects between $4-10 \%$ of the population [1]. Overt hypothyroidism is diagnosed when low levels of the thyroid hormones result in elevated levels of thyroid-stimulating hormone (TSH) above $4.0 \mathrm{mU} / \mathrm{L}$ whereas, subclinical hypothyroidism is diagnosed when TSH levels are elevated above the upper limit of the assay reference range with normal thyroid hormone levels. Thyroid hormones play a paramount role in the normal function of the heart and vascular physiology. Thus, hypothyroidism produces significant cardiovascular effects. Hypothyroidism is known to affect cardiac contractility, which is often diastolic in nature and can lead to decreased cardiac output. Similarly, increased systemic vascular resistance, decreased arterial compliance, and atherosclerosis are common

pathophysiological changes that occur in hypothyroidism [3,4]. Although thyroid hormone deficiency, either clinical or subclinical, is an established risk factor for cardiovascular disease, it is unusual to find threevessel coronary artery disease in a middle-aged woman in our setup who had an insignificant family history and no long-standing coronary artery disease risk factors.

On review of the literature, a case to similar ours was reported from Japan, where a 38-year-old woman with hypothyroidism presented with unstable angina and subsequently underwent CABG [5]. Another case was reported from China, where a patient with hypothyroidism, type 2 diabetes mellitus, and hypertension underwent CABG [6]. Similar cases have been reported from Asia, involving men and women both [7,8]. Many studies conducted across the globe revealed a significant association between subclinical hypothyroidism and subsequent development of ischemic heart disease (IHD) [9-11]. A 2017 metaanalysis of 55 cohort studies concluded that hypothyroidism is associated with higher risks of IHD, cardiac mortality, and all-cause mortality when compared with euthyroidism in the general public or in patients with preexisting cardiac disease. It also noted that subclinical hypothyroidism with elevated TSH levels is associated with an increased risk of IHD events, especially in those with values greater than $10 \mathrm{mIU} / \mathrm{L}$, similar to our case [12]. The findings of these studies show that even subclinical hypothyroidism is a risk factor for coronary artery disease, not alone overt hypothyroidism. In contrast, the European Prospective Investigation into Cancer and Nutrition (EPIC)-Norfolk study did not show any increased risk of coronary artery disease [13].

Moreover, thyroxine replacement therapy is considered as the mainstay treatment for overt hypothyroidism. The use of thyroid replacement therapy can accelerate the development of ischemic heart disease or aggravate the progression of an already existing cardiac disease. This could be due to increased cardiac contractility and improved metabolism, which eventually leads to oxygen supply-demand mismatch resulting in myocardial ischemia and possibly myocardial infarction. One such case was reported in Japan, where the administration of levothyroxine aggravated the patient's clinical condition [5]. Similarly, Flynn et al. reported a population-based study of patients with elevated TSH levels (defined as greater than $4 \mathrm{mIU} / \mathrm{L}$ ) 
and treated with levothyroxine presented with greater risk for cardiovascular events [14]. In another study, no evidence was found to suggest clinically meaningful differences in the pattern of long term health outcomes including all-cause mortality, heart failure, IHD, stroke/transient ischemic attack, and atrial fibrillation in patients on thyroid replacement therapy when TSH concentrations were within normal limits and thus, further emphasizing the need of randomized controlled trials of levothyroxine treatment examining vascular and cardiovascular outcomes [15]. Hence, it is crucial to conduct large scale studies aimed at investigating possible risk factors including thyroid replacement therapy that lead to the development of coronary artery disease in patients with subclinical or overt hypothyroidism, especially in our locality where there is a scarcity of data available on this subject.

\section{Conclusions}

Thyroid hormones play an important role in regulating the heart and vascular physiology. Hypothyroidism, either overt or subclinical, is associated with significant cardiovascular effects. Three vessel disease is a relatively uncommon cardiovascular manifestation of hypothyroidism as most patients present with diastolic dysfunction. Thyroid replacement therapy can accelerate the development of cardiovascular disease or aggravate the underlying heart conditions according to some reports. Therefore, more studies should be conducted to investigate and evaluate the risk factors in hypothyroid patients that can lead to the development of coronary artery disease.

\section{Additional Information \\ Disclosures}

Human subjects: Consent was obtained by all participants in this study. Conflicts of interest: In compliance with the ICMJE uniform disclosure form, all authors declare the following: Payment/services info: All authors have declared that no financial support was received from any organization for the submitted work. Financial relationships: All authors have declared that they have no financial relationships at present or within the previous three years with any organizations that might have an interest in the submitted work. Other relationships: All authors have declared that there are no other relationships or activities that could appear to have influenced the submitted work.

\section{References}

1. Klein I, Danzi S: Thyroid disease and the heart. Circulation. 2007, 116:1725-1735. 10.1161/CIRCULATIONAHA.106.678326

2. Pearce SHS, Brabant G, Duntas LH, Monzani F, Peeters RP, Razvi S, Wemeau JL: 2013 ETA guideline: management of subclinical hypothyroidism. Eur Thyroid J. 2013, 2:215-218. 10.1159/000356507

3. Kahaly GJ, Dillmann WH: Thyroid hormone action in the heart. Endocr Rev. 2005, 26:704-728. 10.1210/er.2003-0033

4. Cooper DS, Biondi B: Subclinical thyroid disease. Lancet. 2012, 379:1142-1154. 10.1016/S01406736(11)60276-6

5. Taguchi T, Iwasaki Y, Asaba K, Takao T, Hashimoto K: Myxedema coma and cardiac ischemia in relation to thyroid hormone replacement therapy in a 38-year-old Japanese woman. Clin Ther. 2007, 29:2710-2714. 10.1016/j.clinthera.2007.12.025

6. Zhang YY, YE JC, Lei WE, Zhang SJ: Coronary artery bypass grafting in a patient with history of esophagectomy, hypothyroidism, hypertension and type 2 diabetes mellitus: a case report. Chin Med J (Engl). 2012, 125:2062-2064. 10.3760/cma.j.issn.0366-6999.2012.11.039

7. Hirano Y, Matsumoto Y, Endoh M, Kasashima F, Abe Y, Sasaki H: Off-pump coronary artery bypass grafting for unstable angina pectoris combined with hypothyroidism and chronic renal failure; report of a case. Kyobu Geka. 2002, 55:799-802.

8. Furukawa K, Ooteki H, Doi K, Shiraishi R: A case report on successful coronary artery bypass grafting (CABG) for angina pectoris combined with hypothyroidism (Article in Japanese). Kyobu Geka. 1997, 50:275278 .

9. Tseng FY, Lin WY, Lin CC, Lee LT, Li TC, Sung PK, Huang KC: Subclinical hypothyroidism is associated with increased risk for all-cause and cardiovascular mortality in adults. J Am Coll Cardiol. 2012, 60:730-737. 10.1016/j.jacc.2012.03.047

10. Imaizumi M, Akahoshi M, Ichimaru S, et al.: Risk for ischemic heart disease and all-cause mortality in subclinical hypothyroidism. J Clin Endocrinol Metab. 2004, 89:3365-3370. 10.1210/jc.2003-031089

11. Razvi S, Weaver JU, Vanderpump MP, Pearce SH: The incidence of ischemic heart disease and mortality in people with subclinical hypothyroidism: reanalysis of the Whickham Survey cohort. J Clin Endocrinol Metab. 2010, 95:1734-1740. 10.1210/jc.2009-1749

12. Ning Y, Cheng YJ, Liu LJ, et al.: What is the association of hypothyroidism with risks of cardiovascular events and mortality? A meta-analysis of 55 cohort studies involving 1,898,314 participants. BMC Med. 2017, 15:21. Accessed: October 21, 2019: 10.1186/s12916-017-0777-9

13. Boekholdt SM, Titan SM, Wiersinga WM, et al.: Initial thyroid status and cardiovascular risk factors: the EPIC-norfolk prospective population study. Clin Endocrinol (Oxf). 2010, 72:404-410. 10.1111/j.13652265.2009.03640.x

14. Flynn RW, Bonellie SR, Jung RT, MacDonald TM, Morris AD, Leese GP: Serum thyroid-stimulating hormone concentration and morbidity from cardiovascular disease and fractures in patients on long-term thyroxine therapy. J Clin Endocrinol Metab. 2010, 95:186-193. 10.1210/jc.2009-1625

15. Thayakaran R, Adderley NJ, Sainsbury C, et al.: Thyroid replacement therapy, thyroid stimulating hormone concentrations, and long term health outcomes in patients with hypothyroidism: longitudinal study. BMJ. 


\section{Cureus}

2019, 366:I4892. Accessed: October 21, 2019: 10.1136/bmj.14892 